\title{
Application of the Similarity Criteria for Estimation of Electromagnetic Compatibility of Frequency Electro Drives with Power Supply System of Industrial Enterprises
}

\author{
Michael A. Averbukh*a, \\ Stanislav V. Khvorostenko a and Evgenia Yu. Sizganovab \\ ${ }^{a}$ Belgorod State Technological University \\ named after V.G. Shukhov \\ 46 Kostyukova Str., Belgorod, 308012, Russia \\ ${ }^{b}$ Siberian Federal University \\ 79 Svobodny, Krasnoyarsk, 660041, Russia
}

Received 04.06.2018, received in revised form 11.09.2018, accepted 03.11.2018

The paper presents similarity theory used for of complex systems for physical modeling mode of operation of variable frequency drives as generators of higher harmonics of currents and voltages in the system of power supply of the enterprise. The $\pi$-criteria of similarity are compiled and scaling factors for a low-power variable frequency electric drive that is in located specific accordance with an industrial variable frequency electric drives of various power. The received criteria and scale factors allowed to estimate electromagnetic compatibility in power supply systems by the example of a factory for the manufacture of reinforced concrete products. The article was prepared within a development program of the Base University on the basis of BSTU named after V.G. Shukhov.

Keywords: similarity theory, $\pi$-criteria, scale coefficient, the similarity of complex systems, variable frequency drive, physical modeling, higher harmonic components of the current and voltage.

Citation: Averbukh M.A., Khvorostenko S.V., Sizganova E.Yu. Application of the similarity criteria for estimation of electromagnetic compatibility of frequency electro drives with power supply system of industrial enterprises, J. Sib. Fed. Univ. Eng. technol., 2018, 11(7), 856-866. DOI: 10.17516/1999-494X-0100.

(c) Siberian Federal University. All rights reserved

This work is licensed under a Creative Commons Attribution-NonCommercial 4.0 International License (CC BY-NC 4.0).

* Corresponding author E-mail address: avers45@rambler.ru, seu_eset@mail.ru 


\title{
Применение критериев подобия
}

\section{для оценки электромагнитной совместимости \\ частотных электроприводов \\ с системой электроснабжения \\ промышленных предприятий}

\author{
М.А. Авербух $x^{a}$, \\ С.В. Хворостенко ${ }^{a}$, Е.Ю. Сизганова ${ }^{\sigma}$ \\ ${ }^{a}$ Белгородский государственный технологический университет \\ им. В.Г. Шухова \\ Россия, 308012, Белгород, ул. Костюкова, 46 \\ ${ }^{6}$ Сибирский федеральный университет \\ Россия, 660041, Красноярск, пр. Свободный, 79
}

\begin{abstract}
В статье представлено применение теории подобия сложных систем для физического моделирования режимов работы частотных электроприводов как генераторов высших гармоник токов и напряжений в систему электроснабжения предприятия. Составленьл л-критерии подобия и масштабные коэффициенты для частотного электропривода малой мощности, находящегося в определенном соответствии с промымленными частотными электроприводамиразличныхмощностей. Полученныекритерииимасштабныекоэффициенты позволили оценить электромагнитную совместимость в системах электроснабжения на примере завода по изготовлению железобетонных изделий. Статья подготовлена в рамках программы развития опорного университета на базе БГТУ им. В.Г. Шухова.
\end{abstract}

Ключевые слова: теория подобия, л-критерии, маситабные коэффициенты, подобие сложных систем, частотно-регулируемый электропривод, физическое моделирование, высиие гармонические составляющие тока и напряжения.

На современных промышленных предприятиях изготовление железобетонных изделий производится поточным способом, где отдельные операции выполняются циклично и изделие перемещается от одного узла к другому. На каждом узле в технологическом процессе производства продукции широко используется частотный регулируемый электропривод (ЧРЭ) на базе полупроводникового преобразователя частоты - асинхронный двигатель с короткозамкнутым ротором (ППЧ-АД) [1], но все они получают питание от одной цеховой подстанции (рис. 1). Так как из-за нелинейности вольтамперных характеристик ЧРЭ является генератором высших гармоник токов и напряжений в цеховую систему электроснабжения, то оценка электромагнитной совместимости ЧРЭ с сетью является актуальной задачей [2]. Подтверждением этому служат проведенные экспериментальные исследования [3] на цеховой подстанции завода ЖБИ, которые показали, что суммарные коэффициенты гармонических составляющих по току $K_{I}$ и напряжению $K_{U}$ на низкой стороне понижающего трансформатора составили: $K_{I}=22 \div 45 \%$, $K_{U}=2.5 \div 9 \%$.

Анализ существующих методов оценки негативного влияния высших гармоник показал, что аналитические методы с использованием схем замещений, имитационных моделей сложны и возможны только при определенных допущениях и упрощениях; эксперименты

$$
-857-
$$




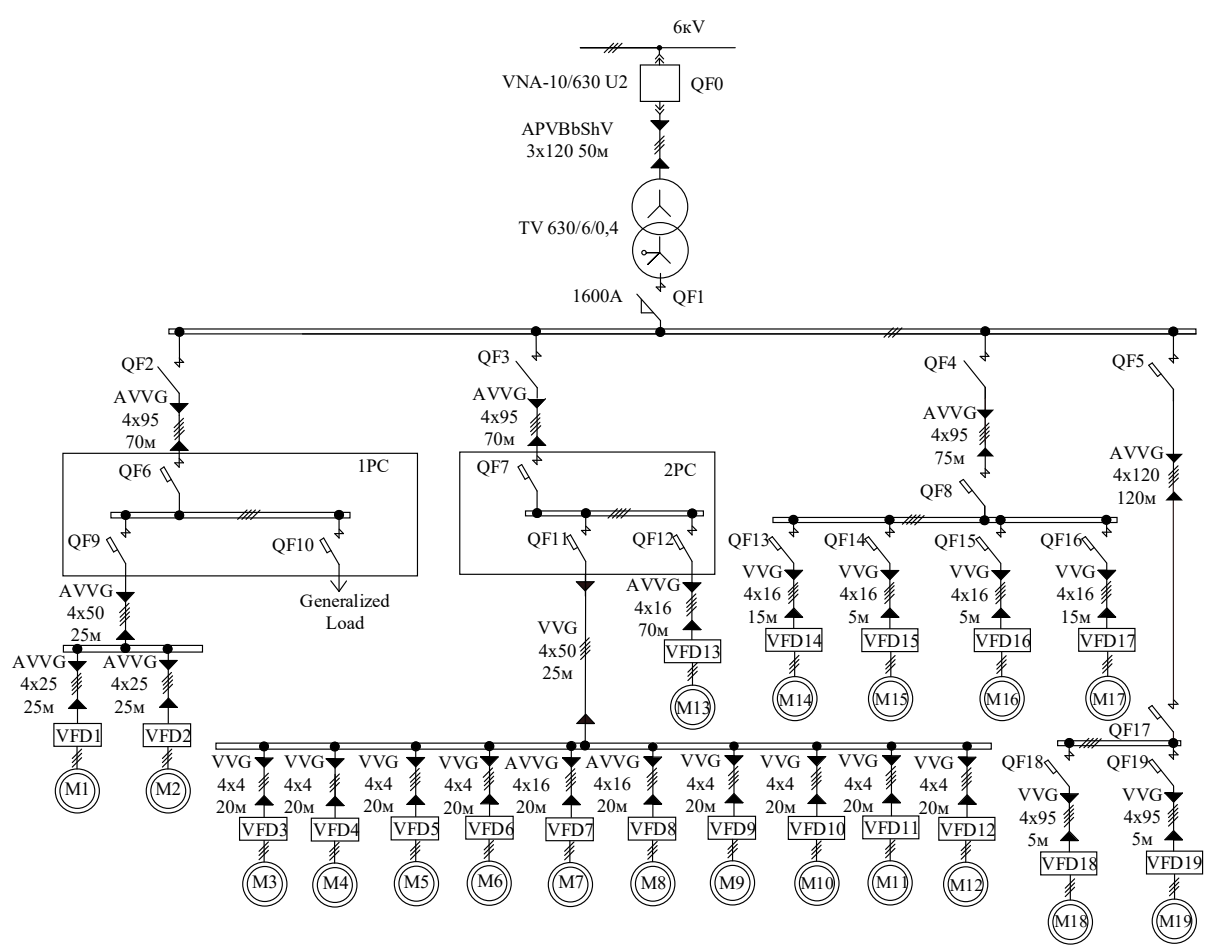

Рис. 1. Схема системы электроснабжения промышленного предприятия по производству железобетонных изделий

Fig. 1. Scheme of the power supply system of an industrial enterprise for the production of reinforced concrete products

позволяют получать результаты с высокой степенью достоверности, но они фиксируют ситуацию только на момент измерений и при определенном режиме данного технологического процесса; физическое моделирование представляет собой метод исследования свойств определенного объекта посредством изучения свойств другого объекта, более удобного для решения задач исследования и находящегося в определенном соответствии (подобии) с первым объектом.

При решении задач в общем случае под физическим моделированием понимается изучение моделируемого объекта, базирующегося на взаимно-однозначном соответствии определенной части свойств оригинала и замещающего его при исследовании объекта, и включающее в себя построение модели, изучение ее и перенос полученных сведений на моделируемый объекторигинал [4].

Суть метода физического моделирования основывается на теории подобия, которая представлена тремя теоремами. Основные положения этих теорем определяют свойства подобных объектов исследования и указывают требования, при удовлетворении которых один из объектов может рассматриваться как модель по отношению к остальным. Основной характеристикой подобных объектов являются критерии подобия, с помощью которых устанавливается взаимооднозначное соответствие модели и оригинала. Критерии подобия - это идентичные по форме алгебраической записи для подобных объектов безразмерные степенные комплексы определенных групп параметров, характеризующих эти объекты [5]. 


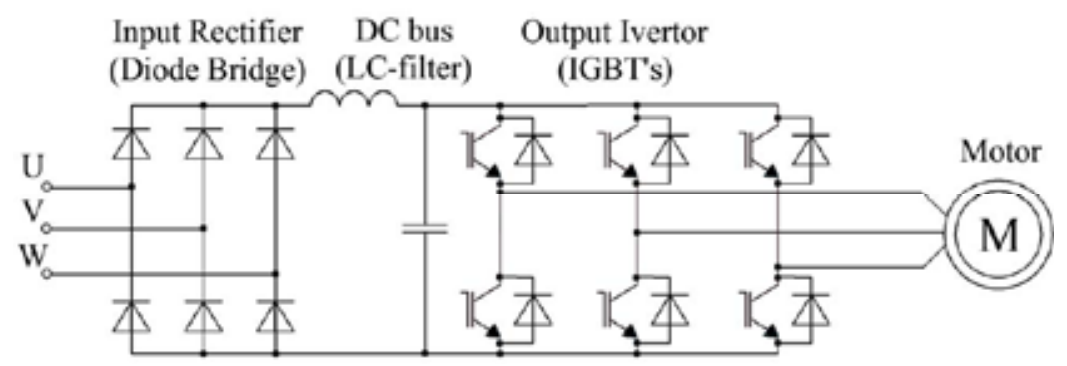

Рис. 2. Силовая часть принципиальной схемы частотно-регулируемого электропривода

Fig. 2. Power circuit diagram variable frequency drive motor

В качестве объектов подобия принята силовая часть принципиальной схемы ЧРЭ (рис. 2) как источника высших гармонических составляющих тока и напряжения. Правомерность использования критериев подобия подтверждается соответствием динамических процессов в ЧРЭ малой и большой мощности. Исходными при описании физических процессов в ЧРЭ являются дифференциальные уравнения отдельных элементов силовой части: неуправляемого выпрямителя (НВ), промежуточного звена постоянного тока (ПЗПТ), автономного инвертора напряжения (АИН) и асинхронного двигателя с короткозамкнутым ротором (АДсКЗ), отражающие интересующие информационные свойства объекта.

Согласно первой теореме для составления $\pi$-критериев подобия параметров применим метод структурного моделирования. Выделив в качестве отдельных элементов ТV, АИН, НВ, ПЗПТ и АДсКЗ, запишем уравнения напряжений в дифференциальной форме для трансформатора и обобщенную систему уравнений каждого элемента двухзвенного преобразователя частоты в системе подвижных координат [u;v], являющихся основополагающими для объектаоригинала [6].

$$
\begin{aligned}
& u_{1}=r_{1} \cdot i_{1}+L_{11} \cdot \frac{d i_{1}}{d t}+L_{12} \cdot \frac{d i_{2}}{d t} \\
& u_{2}=-r_{2} \cdot i_{2}-L_{22} \cdot \frac{d i_{2}}{d t}-L_{21} \cdot \frac{d i}{d t} \text {; } \\
& f_{u}=\frac{u_{u}^{*}}{2 \cdot U_{0}}, f_{v}=\frac{u_{v}^{*}}{2 \cdot U_{0}}, u_{u u}=u_{u} \cdot f_{u}, u_{u v}=u_{u} \cdot f_{v}, i_{u}=\frac{3}{2}\left(i_{1 u} \cdot f_{u}+i_{1 v} \cdot f_{v}\right) \text {; } \\
& u_{u}=u_{B u}, u_{v}=u_{B v}, f_{B u}=\frac{2 \cdot \sqrt{3}}{\pi} \cos \theta_{i}, f_{B v}=\frac{2 \cdot \sqrt{3}}{\pi} \sin \theta_{i} ; \\
& u_{B}=\frac{3}{2}\left(u_{B u} \cdot f_{B u}+u_{B v} \cdot f_{B v}\right), i_{u}=i_{B} \cdot f_{B u}, i_{v}=i_{B} \cdot f_{B v} \text {; } \\
& \frac{d i_{B}}{d t}=\frac{u_{B}-u_{u}}{L_{p \phi}}, \frac{d u_{u}}{d t}=\frac{i_{C}}{C_{6 \phi}}, i_{c}=i_{B}-i_{u} \text {; } \\
& u_{1 u}=\frac{d \psi}{d t}-\omega_{k} \psi{ }_{1 v}+R_{1} i_{1 u}, u_{1 v}=\frac{\mathrm{d} \psi_{1 \mathrm{v}}}{\mathrm{dt}}-\omega_{\mathrm{k}} \psi_{1 \mathrm{u}}+\mathrm{R}_{1} \mathrm{i}_{1 \mathrm{v}}, \mathrm{J} \frac{\mathrm{d} \omega_{\ni}}{\mathrm{dt}}=\mathrm{M}-\mathrm{M}_{\mathrm{c}} \text {; } \\
& 0=\frac{\mathrm{d} \psi_{2 \mathrm{u}}}{\mathrm{dt}}-\omega_{\mathrm{k}} \psi_{2 v}-\omega_{\ni} \psi_{2 v}+\mathrm{R}_{2} \mathrm{i}_{2 \mathrm{u}}, 0=\frac{\mathrm{d} \psi_{2 v}}{\mathrm{dt}}+\omega_{\mathrm{k}} \psi_{2 \mathrm{u}}-\omega_{\ni} \psi_{2 \mathrm{u}}+\mathrm{R}_{2} \mathrm{i}_{2 \mathrm{v}} \text {; } \\
& \mathrm{M}=\frac{3}{2} \mathrm{p}_{\Pi} \psi_{0 \mathrm{u}} \mathrm{i}_{1 v}-\frac{3}{2} \mathrm{p}_{\Pi} \psi_{0 v} \mathrm{i}_{1 \mathrm{u}},
\end{aligned}
$$


где $u_{1}, i_{1}$ - мгновенные значения напряжения и тока первичной обмотки; $u_{2}, i_{2}-$ мгновенные значения напряжения и тока вторичной обмотки, приведенные к первичной; $r_{1}, r_{2}, L_{11}, L_{22}-$ активные сопротивления и собственные индуктивности обмоток; $L_{12}, L_{21}$ - взаимная индуктивность обмоток; $u_{u}^{*}, u_{v}^{*}$ - преобразованные задающие воздействия; $f_{u}, f_{v}-$ усредненные коммутационные функции (индекс модуляции); $U_{0}$ - амплитуда опорного сигнала; $u_{u}-$ напряжение источника питания инвертора; $i_{u}$ - усредненный ток питания инвертора; $u_{u u}, u_{u v}-$ усредненные выходные напряжения инвертора; $i_{l u}, i_{l v}$ - усредненные выходные токи инвертора; $u_{u}, u_{v}$, $i_{u}, i_{v}$ - преобразованные основные гармоники напряжений на силовом входе неуправляемого выпрямителя и токов сети; $f_{\text {вu }}, f_{\text {вv }}$ - преобразованные основные гармоники коммутационных функций неуправляемого выпрямителя; $\theta_{i}$ - угол поворота обобщенного вектора коммутационной функции выпрямителя, или результирующего вектора тока сети, относительно оси фазы А напряжения сети; $u_{b}, i_{b}$ - напряжение и ток на выходе выпрямителя; $L_{p \phi}-$ индуктивность сглаживающего реактора LC-фильтр; $C_{б \phi}-$ емкость конденсаторной батареи фильтра; $i_{c}$ - ток конденсатора фильтра; $u_{1 u}, u_{1 v}, i_{l u}, i_{l v}, \psi_{l u}, \psi_{l v}$ - соответственно преобразованные напряжения, токи и полные потокосцепления обмотки статора; $i_{2 u}, i_{2 v}, \psi_{2 u}, \psi_{2 v}-$ преобразованные токи и полные потокосцепления обмотки ротора; $\psi_{0 u}, \psi_{0 v}-$ главное потокосцепление; $p_{n}$ - число пар полюсов.

На основе теоремы о подобии сложных систем, состоящих из подсистем, и используя правило Фурье, определим критерии подобия путем приведения уравнений к безразмерному виду, а именно способом интегральных аналогов [4]. При этом сопоставляемые процессы в объекте-оригинале и объекте-подобии подобны, поэтому между их сходственными параметрами должны существовать соотношения пропорциональности, например для ПЗПТ (уравнение 1), (параметры модели подобия обозначаются теми же буквами, но со штрихами):

$$
i_{c}=m_{i_{c}} \cdot i_{c}^{\prime} ; \quad i_{B}=m_{i_{B}} \cdot i_{B}^{\prime} ; \quad i_{u}=m_{i_{u}} \cdot i_{u}^{\prime},
$$

где $m_{i}$ - масштабные коэффициенты.

Подобие процессов в оригинале и объекте-подобии означает, что они, имея качественно одинаковый характер и различаясь лишь масштабами, должны описываться одинаковыми математическими уравнениями. Это возможно только в том случае, если будут равны единице комбинации масштабных коэффициентов при соответствующих членах однородного уравнения, например для ПЗПТ:

$$
\begin{aligned}
& \mathrm{I}_{\pi 1}=\frac{\mathrm{u}_{b} \cdot \mathrm{t} \cdot \mathrm{L}_{p \phi}^{\prime} \cdot \mathrm{i}_{B}^{\prime}}{\mathrm{u}_{B}^{\prime} \cdot \mathrm{t}^{\prime} \cdot \mathrm{L}{ }_{p \phi} \cdot \mathrm{i}_{B}}=1 ; \quad \mathrm{I}_{\pi 2}=\frac{\mathrm{u}_{u} \cdot \mathrm{t} \cdot \mathrm{L}_{p \phi}^{\prime} \cdot \mathrm{i}_{B}^{\prime}}{\mathrm{u}_{u}^{\prime} \cdot \mathrm{t}^{\prime} \cdot \mathrm{L}_{p \phi} \cdot \mathrm{i}_{B}}=1 ; \\
& \mathrm{I}_{\pi 3}=\frac{\mathrm{i}_{c} \cdot \mathrm{t} \cdot \mathrm{C}_{\delta \phi}^{\prime} \cdot \mathrm{u}_{u}^{\prime}}{\mathrm{i}_{c}^{\prime} \cdot \mathrm{t}^{\prime} \cdot \mathrm{C}_{\delta \phi} \cdot \mathrm{u}_{u}}=1 ; \mathrm{I}_{\pi 4}=\frac{\mathrm{i}_{b} \cdot \mathrm{i}_{c}^{\prime}}{\mathrm{i}_{b}^{\prime} \cdot \mathrm{i}_{c}}=1 ; \mathrm{I}_{\pi 5}=\frac{{ }^{\mathrm{i}_{u} \cdot \mathrm{i}_{c}^{\prime}}}{\mathrm{i}_{u}^{\prime} \cdot \mathrm{i}_{c}}=1 .
\end{aligned}
$$

Критерии подобия трансформатора и ЧРЭ (по правилу интегральных аналогов) получены путем приведения уравнений к безразмерному виду:

- трансформатор: 


$$
\begin{aligned}
& \pi_{1}=\frac{L_{11} \cdot i_{1}}{r_{1} \cdot i_{1} \cdot t} ; \quad \pi_{2}=\frac{L_{12} \cdot i_{2}}{r_{1} \cdot i_{1} \cdot t} ; \pi_{3}=\frac{u_{1}}{r_{1} \cdot i_{1}} ; \\
& \pi_{4}=\frac{-L_{22} \cdot i_{2}}{r_{2} \cdot i_{2} \cdot t} ; \quad \pi_{5}=\frac{-L_{21} \cdot i_{1}}{r_{2} \cdot i_{2} \cdot t} ; \pi_{6}=\frac{-u_{2}}{r_{2} \cdot i_{2}} ;
\end{aligned}
$$

- АИН:

$$
\begin{aligned}
& \pi_{7}=\frac{0.5 \cdot u_{u}^{*}}{U_{0} \cdot f_{u}} ; \quad \pi_{8}=\frac{0.5 \cdot u_{v}^{*}}{U_{0} \cdot f_{v}} ; \quad \pi_{9}=\frac{u_{\mathrm{и}} \cdot f_{u}}{u_{\text {и } u}} ; \\
& \pi_{10}=\frac{u_{\text {и }} \cdot f_{v}}{u_{\text {и }}} ; \quad \pi_{11}=\frac{1.5 \cdot i_{1 u} \cdot f_{u}}{i_{\text {и }}} ; \quad \pi_{12}=\frac{1.5 \cdot i_{1 v} \cdot f_{v}}{i_{\text {и }}} ;
\end{aligned}
$$

- HB:

$$
\begin{aligned}
& \pi_{13}=\frac{u_{\mathrm{B} u}}{u_{u}} ; \pi_{14}=\frac{u_{\mathrm{B} v}}{u_{v}} ; \pi_{15}=\frac{1.103 \cdot \cos \theta}{f_{\mathrm{B} u}} ; \pi_{16}=\frac{1.103 \cdot \sin \theta}{f_{\mathrm{B} v}} ; \\
& \pi_{17}=\frac{1.5 \cdot u_{\mathrm{B} u} \cdot f_{\mathrm{B} u}}{u_{\mathrm{B}}} ; \pi_{18}=\frac{1.5 \cdot u_{\mathrm{B} v} \cdot f_{\mathrm{B} v}}{u_{\mathrm{B}}} ; \pi_{19}=\frac{i_{\mathrm{B}} \cdot f_{\mathrm{B} u}}{i_{u}} ; \pi_{20}=\frac{i_{B} \cdot f_{B v}}{i_{v}} ;
\end{aligned}
$$

- $\quad$ ПЗПТ:

$$
\pi_{21}=\frac{u_{\mathrm{B}} \cdot t}{L_{\mathrm{p \phi}} \cdot i_{\mathrm{B}}} ; \quad \pi_{22}=\frac{u_{\mathrm{u}} \cdot t}{L_{\mathrm{p \phi}} \cdot i_{\mathrm{B}}} ; \quad \pi_{23}=\frac{i_{\mathrm{c}} \cdot t}{C_{\text {бф }} \cdot u_{\mathrm{u}}} ; \pi_{24}=\frac{i_{\mathrm{H}}}{i_{c}} ; \pi_{25}=\frac{i_{\mathrm{B}}}{i_{c}} ;
$$

- АД:

$$
\begin{aligned}
& \pi_{26}=\frac{\psi_{1 u}}{R_{1} i_{1 u} t} ; \quad \pi_{27}=\frac{u_{1 u}}{R_{1} i_{1 u}} ; \pi_{28}=\frac{\psi_{1 v}}{R_{1} i_{1 v} t} ; \quad \pi_{29}=\frac{u_{1 v}}{R_{1} i_{1 v}} ; \\
& \pi_{30}=\frac{\psi_{2 u}}{R_{2} i_{2 u} t} ; \quad \pi_{31}=\frac{\omega_{3} \psi_{2 v}}{R_{2} i_{2 u}} ; \pi_{32}=\frac{\psi_{2 v}}{R_{2} i_{2 v} t} ; \quad \pi_{33}=\frac{\omega_{\ni} \psi_{2 u}}{R_{2} i_{2 v}} ; \\
& \pi_{34}=\frac{p_{n} \psi_{0 u} i_{1 v}}{M} ; \pi_{35}=\frac{p_{n} \psi_{0 v} i_{1 u}}{M} ; \quad \pi_{36}=\frac{J \omega_{\ni}}{M t} ; \pi_{37}=\frac{M_{c}}{M} .
\end{aligned}
$$

Подобие сложных систем (ЧРЭ), состоящих из подсистем (НВ, ПЗПТ, АИН и АД), соответственно подобных в отдельности, обеспечивается подобием всех сходных элементов и связей, являющихся общими для этих подсистем.

Рассмотрим два электропривода на базе ППЧ-АД (ATV71U22M3-АИР80B4Б01 и VFD1100CH43A-4A160S4У3), технические характеристики и параметры представлены в табл. 1.

На рис. 3 представлены механические характеристики ЧРЭ, полученные опытным путем при частотах 30; 40; 50 Гц, на выходе АИН: a) - ATV71U22M3 b) - VFD1100CH43A(I) и с учетом масштабных коэффициентов $\mathrm{m}_{\omega}=2.081, \mathrm{~m}_{\mathrm{M}}=35.24$ и критериев подобия (II).

Как следует из рис. $3 b$, механические характеристики ЧРЭ, полученные в результате применения критериев подобия, показали хорошую сходимость с экспериментальными механическими характеристиками, что говорит о правомерности использования критериев подобия для оценки динамических процессов ЧРЭ различных мощностей. Таким образом, использование рассмотренной методики позволяет на основании исследуемого образца (оригинала) пере- 
Michael A. Averbukh, Stanislav V. Khvorostenko... Application of the Similarity Criteria for Estimation of Electromagnetic...

Таблица 1. Технические характеристики и параметры частотно-регулируемого электропривода

Table 1. Technical characteristics and parameters variable frequency drive motor

\begin{tabular}{|c|c|c|c|c|c|}
\hline Тип АД & АИР80В4Б01 & $4 \mathrm{~A} 280 \mathrm{~S} 2 \mathrm{Y} 3$ & Тип ППЧ & ATV71U22M3 & VFD1100CH43A \\
\hline $\mathrm{P}$, кВт & 1.5 & 110 & $\mathrm{~S}$, кВA & 3.5 & 167 \\
\hline $\mathrm{n}_{0}$, об/мин & 1435 & 2970 & $\mathrm{U}_{\mathrm{л}}, \mathrm{B}$ & 380 & 380 \\
\hline$\psi_{1 \mathrm{u}}$ & 0.0626 & 0.218 & $\mathrm{I}_{\text {ном}}, \mathrm{A}$ & 3.7 & 220 \\
\hline$\psi_{10}$ & -0.903 & -0.967 & $\mathrm{u}_{\mathrm{u}}, \mathrm{B}$ & 589.8 & 589.8 \\
\hline $\mathrm{R}_{1}, \mathrm{OM}_{\mathrm{M}}$ & 7.403 & 0.05 & $\mathrm{i}_{\mathrm{c}}, \mathrm{A}$ & 3.77 & 290.1 \\
\hline $\mathrm{i}_{1 \mathrm{u}}, \mathrm{A}$ & 3.7 & 147.984 & $\mathrm{C}_{\text {бф}}$, мкФ & 620 & 6100 \\
\hline $\mathrm{i}_{1 v}, \mathrm{~A}$ & -2.66 & -13.66 & $\mathrm{~L}_{\mathrm{p} \phi}, \mathrm{M} \Gamma_{\mathrm{H}}$ & 0.804 & 0.042 \\
\hline $\mathrm{u}_{\mathrm{lu}}, \mathrm{B}$ & 311 & 311 & & & \\
\hline $\mathrm{u}_{1 v}, \mathrm{~B}$ & 311 & 311 & & & \\
\hline$\psi_{2 \mathrm{u}}$ & -0.081 & 0.117 & & & \\
\hline$\psi_{20}$ & -0.854 & 0.012 & & & \\
\hline $\mathrm{R}_{2}, \mathrm{OM}_{\mathrm{M}}$ & 4.257 & 0.027 & & & \\
\hline $\mathrm{i}_{2 \mathrm{u}}, \mathrm{A}$ & -3.69 & -133 & & & \\
\hline $\mathrm{i}_{2 v}, \mathrm{~A}$ & 0.349 & 1324 & & & \\
\hline$\psi_{0 \mathrm{u}}$ & 0.006 & 0.176 & & & \\
\hline$\psi_{0 v}$ & -0.86 & -0.572 & & & \\
\hline $\mathrm{p}_{\text {п }}$ & 2 & 1 & & & \\
\hline $\mathrm{t}, \mathrm{c}$ & 1 & 1 & & & \\
\hline $\mathrm{M}, \mathrm{H} \cdot \mathrm{M}$ & 10.1 & 350 & & & \\
\hline $\mathrm{M}_{\mathrm{c}}, \mathrm{H} \cdot \mathrm{M}$ & 7.1 & 245 & & & \\
\hline $\mathrm{J}, \kappa^{\cdot} \mathrm{M}^{2}$ & 0.0033 & 1.1 & & & \\
\hline$\omega_{э}$, рад/с & 157 & 314 & & & \\
\hline
\end{tabular}

носить результаты оценки аналогичных физических процессов ЧРЭ с одного исследуемого электропривода на другой при различных мощностях двигателя, но при одинаковых режимах работы.

Так как процесс генерирования высших гармоник в ЧРЭ не зависит от мощности электропривода, а определяется только параметрами НВ частотного преобразователя, поэтому предлагаемая оценка масштабных коэффициентов и критериев подобия аналогична для оценки основных показателей ЭМС. Инженерный метод расчета составляющих гармонического спектра позволяет определить действующее значение высших гармоник тока и напряжения при работе преобразователя [7]:

$$
\begin{gathered}
I(n)_{i}=\frac{m \cdot S_{\Pi P i}}{\sqrt{3} \cdot \pi \cdot U_{\text {л }} \cdot x_{\sum i} \cdot n^{2}} \cdot \sin \varphi_{i} \cdot \sin \left(\frac{3 \cdot n \cdot x_{\sum i}}{m \cdot \sin \varphi_{i}}\right), \\
U(n)_{i}=\frac{m}{\pi \cdot n} \cdot U_{\text {Л }} \cdot \frac{x_{C i}^{*}}{x_{\sum i}} \cdot \sin \varphi_{i} \cdot \sin \left(\frac{* \cdot n \cdot x_{\sum i}}{m \cdot \sin \varphi_{i}}\right), \\
-862-
\end{gathered}
$$



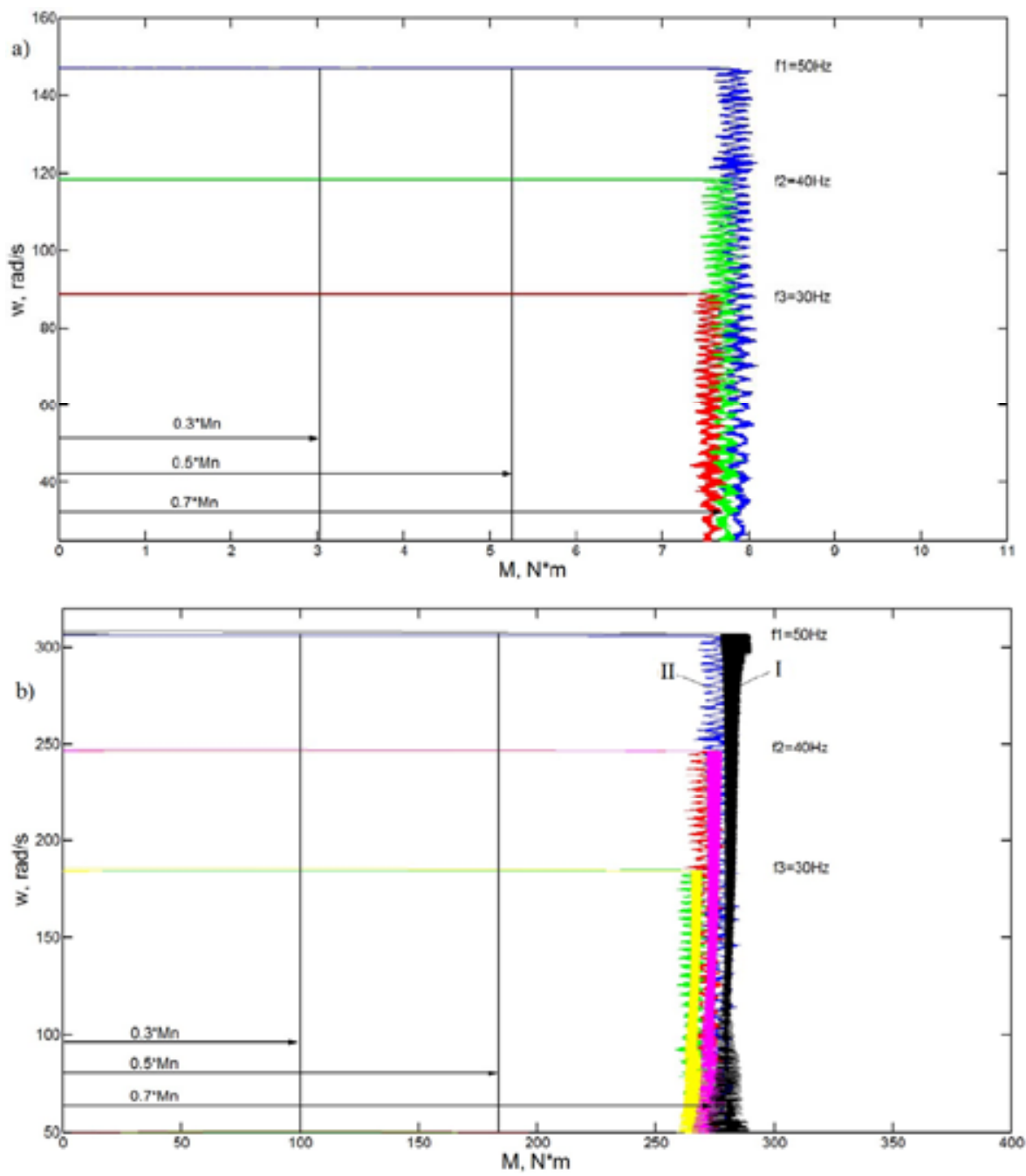

Рис. 3. Механические характеристики частотно-регулируемого электропривода

Fig. 3. Mechanical characteristics of variable frequency drive motor

где $m$ - число фаз выпрямителя $(m=6) ; U_{л}-$ линейное напряжение сети; $x_{\Sigma}-$ суммарное индуктивное сопротивление одной из ветви цеховой системы электроснабжения, в которой есть электроприемник с нелинейной вольтамперной характеристикой; $n$ - номер канонической гармоники; $\sin \varphi_{\mathrm{i}}-$ коэффициент реактивной мощности; $\mathrm{x}_{C i}-$ эквивалентное сопротивление системы в относительных единицах, т.е. сопротивление от условной точки сети бесконечной мощности до точки сети, в которой определяется суммарный коэффициент гармонических составляющих.

Критерии подобия и масштабные коэффициенты определены для действующего значения тока и напряжения любой гармоники в цепи преобразователя (по правилу интегральных аналогов), путем приведения уравнений к безразмерному виду: 


$$
\begin{aligned}
& \pi_{38(i)}=\frac{S_{\Pi P i}}{x_{\Sigma i} \cdot I(n)_{i}} \cdot \sin \left(\frac{3 \cdot n \cdot x_{\sum i}}{m \cdot \sin \varphi_{i}}\right), \\
& \pi_{39(i)}=\frac{1}{U(n)_{i} \cdot x_{\Sigma i}} \cdot \sin \left(\frac{3 \cdot n \cdot x_{\Sigma i}}{m \cdot \sin \varphi_{i}}\right),
\end{aligned}
$$

где $I(n)_{i}, U(n)_{i}$ - действующее значение высшей гармоники тока и напряжения соответственно.

На рис. 4 представлены гистограммы высших гармонических составляющих тока и напряжения с указанными $\mathrm{K}_{\mathrm{I}}$ и $\mathrm{K}_{\mathrm{U}}$ в \%. Красный и синий цвета относятся к ATV71U22M3 и VFD1100CH43A соответственно и получены опытным путем, а желтым цветом отмечены гистограммы, полученные с помощью критериев подобия масштабных коэффициентов для исследовательской модели.

Гистограммы на рис. 4 подтвердили корректность применения критериев подобия и масштабных коэффициентов для оценки высших гармоник, расхождение экспериментальных и критериальных данных не превышает 5 \%.

\section{Выводы}

1. На промышленных предприятиях для реализации технологического процесса широко используются рабочие механизмы с ЧРЭ с широким диапазоном мощностей. При этом если в качестве преобразователя частоты используются полупроводниковые преобразователи с промежуточным звеном постоянного тока, то независимо от типов и установленных мощностей физические процессы по генерированию высших гармоник токов и напряжений идентичны.Поэтому для оценки показателей ЭМС предложено использовать критерии подобия и масштабные коэффициенты, позволяющие на основании экспериментальных, аналитических результатов оценки показателей ЭМС одного электропривода трансформировать результаты на все остальные.

2. Полученные критерии подобия и масштабные коэффициенты на основании однотипной записи дифференциальных уравнений для всех элементов ЧРЭ с использованием трех теорем подобия позволяют исследовать и количественно оценивать весь спектр высших гармоник токов и напряжений, однотипных ЧРЭ с различной установленной мощностью и параметрами питающей сети.

3. Корректность применение критериев подобия и масштабных коэффициентов для оценки показателей ЭМС произведена на серии ЧРЭ различных мощностей. Например, для ЧРЭ с установленной мощностью двигателя $\mathrm{P}_{\mathrm{H}}=1.5$ кВт и $\mathrm{P}_{\mathrm{H}}=110$ кВт были измерены и аналитически рассчитаны показатели ЭМС и уровни высших гармоник токов и напряжений, затем эти же показатели для ЧРЭ с двигателем $\mathrm{P}_{\mathrm{H}}=110$ кВт были определены на основании критериев подобия и масштабных коэффициентов на основе показателей электропривода с двигателем $\mathrm{P}_{\mathrm{H}}=1.5$ кВт. Расхождение результатов между натурными экспериментами и полученными на основании критериев подобия не превысили 5-18 \% для 5-й и 7-й гармоник и $5 \%$ для коэффициентов суммарных гармонических составляющих по току и напряжению. 
a)

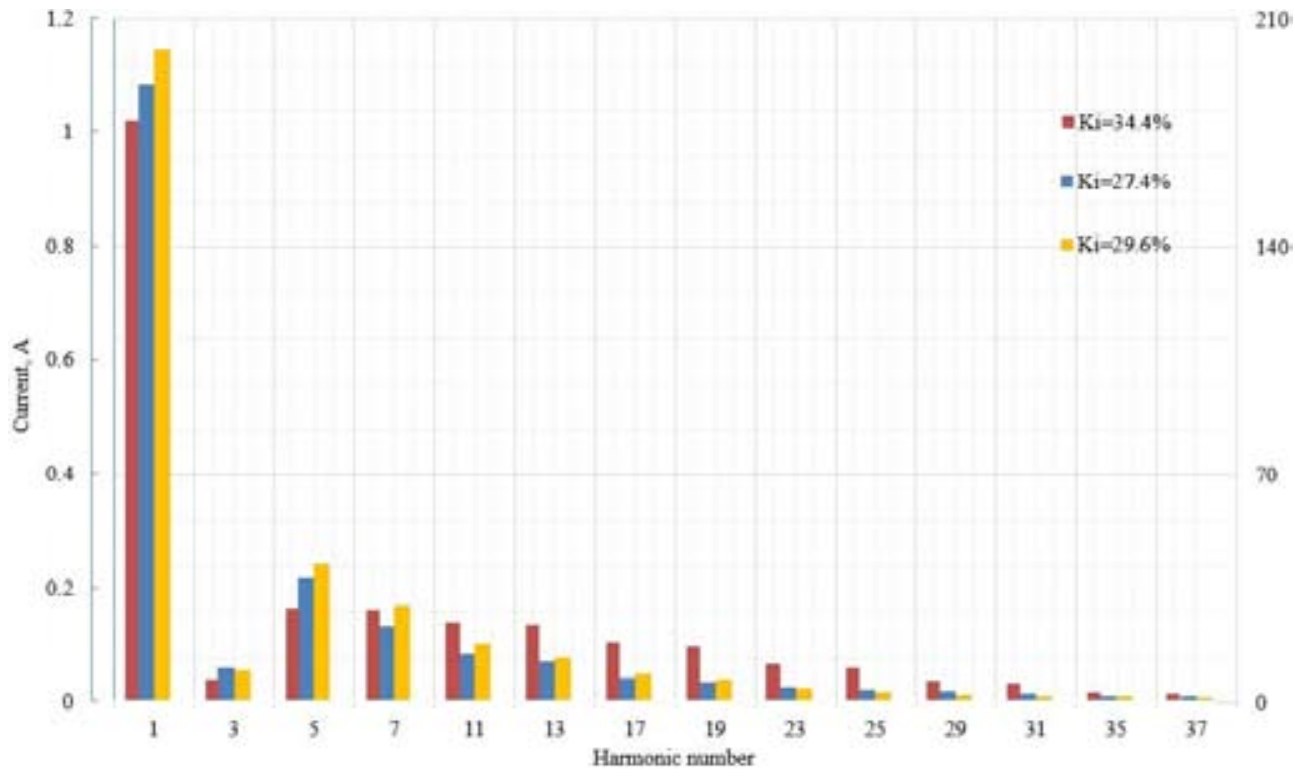

b)

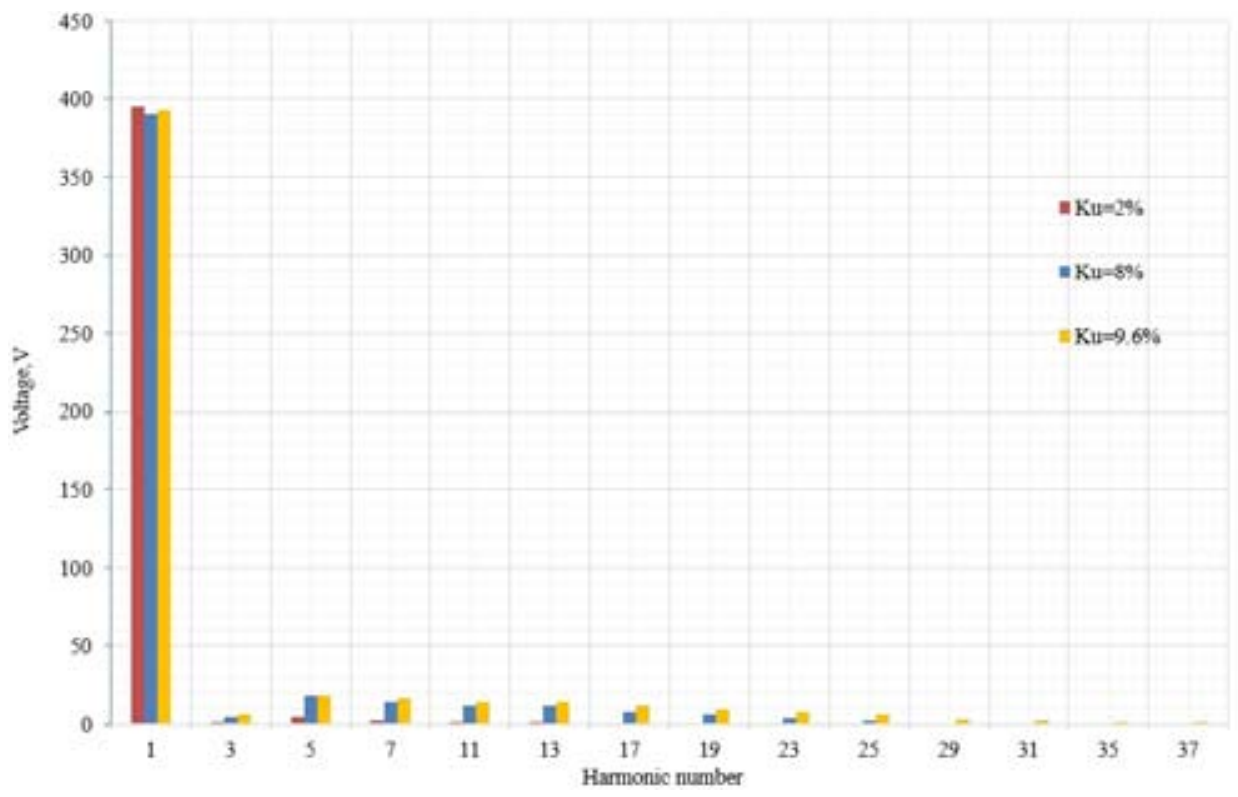

Рис. 4. Гистограмма высших гармоник: а) тока; b) напряжения

Fig.4. Histogram higher harmonics: a) of the current; b) of the voltage

\section{Список литературы}

[1] Боярская Н.П., Довгун В.П., Егоров Д.Э. Синтез фильтрокомпенсирующих устройств для систем электроснабжения. Красноярск: СФУ, 2014, 192 с. [Boyarskaya N.P., Dovgun V.P., Egorov D.E. Synthesis filter-devices for power supply systems. Krasnoyarsk: SFU. 2014, 192 p. (in Russian)] 
[2] Авербух М.А., Коржов Д.Н., Лимаров Д.С. Экспериментальная оценка уровня высших гармоник в схеме электроснабжения трубогибочного стана УЗТМ-465. Промьшленная энергетика, 2015, 1, 48-54. [Averbuh M.A., Korzhov D.N., Limarov D.S. Experimental estimation of the level of higher harmonics in the power supply system of the UZTM-465 pipe bender. Industrial power engineering, 2015, 1, 48-54 (in Russian)]

[3] Авербух М.А., Прасол Д.А., Хворостенко С.В. Экспериментальное исследование несинусоидальных режимов цеховой системы электроснабжения при динамическом вибрационном формировании бетонных смесей. Журнал МГТУ, Электротехнические системы и комплексы, 2017, 1(34), 24-31. [Averbuh M.A., Prasol D.A., Khvorostenko S.V. Experimental study of non-sinusoidal regimes of the shop system of power supply under dynamic vibrational formation of concrete mixtures. J. MGTU, Electrotechnical systems and complexes, 2017, 1(34), 24-31 (in Russian)]

[4] Веников В.А. Теория подобия и моделирования (для энергетики). Изд. 4-е, доп. и перераб. М., 2014, 479 с. [Venikov V.A. Theory of similarity and modeling (for power engineering). Edition 4 iscompletedandrevised. Moscow, 2014, 479 p. (in Russian)]

[5] Josef, Kuneš. Similarity and Modeling in Science and Engineering. Czech Republic: Springer, 2012,49 p.

[6] Браславский И.Я., Ишматов З.Ш., Поляков В.Н. Энергосберегающий асинхронный электропривод. М.: Академия, 2004. 256 с. [Braslavsky I.Y., Ishmatov Z.S., Polyakov V.N. Energysaving asynchronous electric drive. Moscow, Academy, 2004, 256 p. (in Russian)]

[7] Иванов В.С., Соколов В.И. Режимы потребления и качество электроэнергии систем электроснабжения промышленных предприятий. М.: Энергоатомиздат, 1987, 336 c. [Ivanov V.S., Sokolov V.I. Consumption modes and quality of electric power of power supply systems of industrial enterprises. Moscow, Energoatomizdat, 1987, 336 p. (in Russian)] 